\section{Chronische lymphatische Leukämie}

\section{Zytostatika-freie Regime: Alter- native zur Immunchemotherapie}

Bei der chronischen lymphatischen Leukämie (CLL) haben sich Kombinations-Chemotherapien als effektiv erwiesen. Die Wirksamkeit konnte in der Folge durch Addition monoklonaler Antikörper noch gesteigert werden. Mittlerweile wurden neue Substanzen entwickelt, die innovative Kombinationsoptionen eröffnen und eine verbesserte Eradikation der minimalen Resterkrankung (MRD) erlauben. Eine Rückschau auf den ASH 2017.

Bei Patienten mit rezidivierter/refraktärer CLL ( $r$ CLL) haben die Tumorzellen bereits zahlreiche biologische Anomalien entwickelt - das Ansprechen auf die konventionelle Immunchemotherapie ist suboptimal: So beträgt die Responserate bei Bendamustin/Rituximab (BR) als häufigster Rezidivtherapie ca. $60 \%$, die Rate kompletter Remissionen (CR) nur $9 \%$. Auch das mediane progressionsfreie Überleben (PFS) ist mit 15 Monaten recht kurz, berichtete Prof. John F. Seymour, Melbourne/ Australien. Als attraktive Alternative bietet sich die Kombination des antiapoptotisch wirksamen BCL2-Inhibitors Venetoclax mit Rituximab (VenR) an, die in einer Phase-lbStudie bereits durch ein akzeptables Sicherheitsprofil und hohe CR-Raten überzeugte (1).

Dieses Regime wurde jetzt in der Phase-IIIStudie MURANO weiter geprüft: Die Studie umfasste 389 Patienten mit rrCLL, die randomisiert dem Standardarm mit sechs Zyklen BR oder der experimentellen VenR-Kombination zugeteilt wurden (2). Zur Vermeidung eines Tumorlysesyndroms (TLS) wurde Venetoclax innerhalb von fünf Wochen unter sorgfältigem Monitoring bis zu einer Dosis von $400 \mathrm{mg}$ hochtitriert. Bei guter Verträglichkeit konnte der BCL2-Inhibitor bis zu zwei Jahre lang weiter verabreicht werden. $27 \%$ der Patienten waren aufgrund des Vorliegens der 17p-Deletion als Hochrisikokollektiv charakterisiert.

\section{Progressionsfreies und Gesamtüberleben verlängert}

Beim primären Endpunkt, dem durch die Prüfärzte ausgewerteten PFS, überzeugte das VenR-Regime aufgrund der signifikanten Reduktion des Progressionsrisikos um mehr als $80 \%$ : Nach einem Follow-up von knapp zwei Jahren lag das PFS im Kontrollarm bei median
17 Monaten und war damit sogar etwas länger als in früheren Studien, so Seymour. Im experimentellen Arm mit VenR ist dagegen noch weit über die Hälfte der Patienten ohne Progress, der PFS-Median noch nicht erreicht (HR 0,$17 ; p<0,0001$ ) ( Abb. 1). Die 2-Jahres-Rate für das PFS stieg von 36,3\% mit BR auf 84,9\% mit VenR. Das VenR-Regime erwies sich dem bisherigen Standard in allen biologischen und klinischen Subgruppen und auch in der Hochrisikogruppe mit 17p-Deletion, als überlegen.

Die Rate der anhaltend über zwei Jahre MRD-negativen Patienten war mit $60 \%$ ebenfalls hoch. Im Kontrollarm waren nach neun Monaten rund $13 \%$ der Patienten MRD-negativ; die Rate sank jedoch im weiteren Verlauf wieder ab. Selbst nach dem derzeit noch kurzen Follow-up ist auch beim Gesamtüberleben (OS) bereits eine Verbesserung festzustellen, die Seymour als „klinisch relevant" wertete: Die 2-Jahres-Rate beträgt im Standardarm $86,6 \%$, während im VenR-Arm noch 91,9\% der Patienten leben.

Das neue Regime wurde insgesamt gut vertragen. Am häufigsten waren Neutropenien; Infektionen traten nur selten auf. Auch das gefürchtete TLS konnte durch die allmähliche Hochtitration von Venetoclax meist vermieden werden und wurde nur bei $3 \%$ der Patienten beobachtet. „Nach den MURANO-Daten kann das VenR-Regime als neue Standardoption bei der rrCLL betrachtet werden", resümierte Seymour.

\section{Effektive Zytostatika-freie Kombination}

Der Bruton-Tyrosinkinase-Inhibitor Ibrutinib hat bei der CLL bereits eine hohe Wirksamkeit gezeigt. In Studien werden derzeit Kombinationen von Venetoclax und Ibrutinib geprüft, um eine starke MRD-Eradikation zu erreichen und das Outcome von CLL-Patienten weiter zu verbessern. Als Rationale für diese Kombination nannte Dr. Nitin Jain, Houston/USA, die komplementären Wirkmechanismen und die nicht überlappenden Toxizitäten beider Substanzen sowie die präklinisch bereits belegte Synergie.

Seine Arbeitsgruppe prüfte das Zweierregime in einer Phase-II-Studie bei 37 rrCLLPatienten (Kohorte 1) und 40 therapienaiven CLL-Patienten mit mindestens einem Hochrisi- 


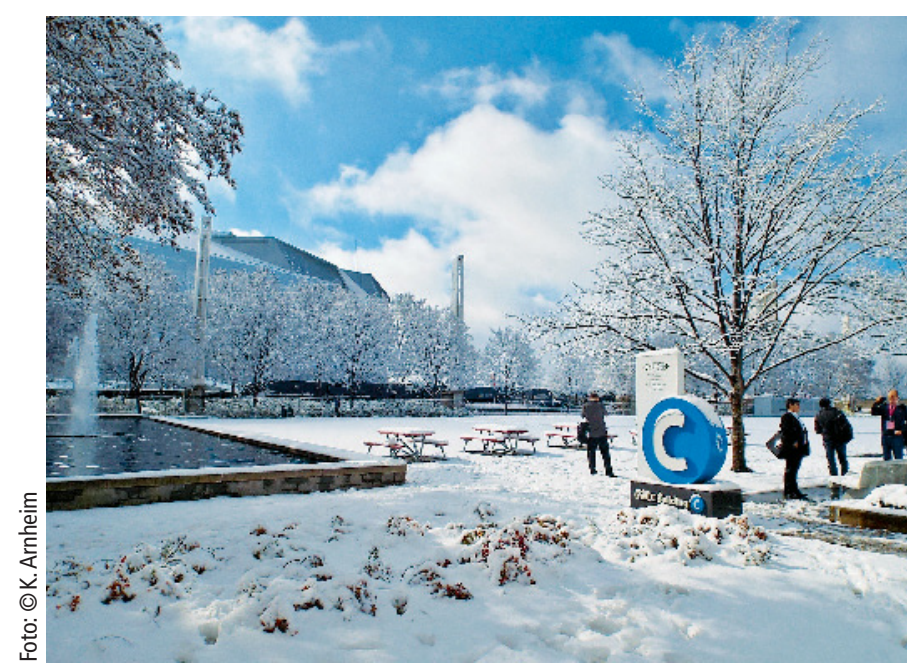

Die ASH-Jahrestagung fand 2017 im winterlich verschneiten Atlanta/USA statt. kofaktor wie 17p- oder 11q-Deletion, unmutiertes IgVH oder mutiertes TP53 (Kohorte 2) (3). Insgesamt besaßen $92 \%$ der Patienten in Kohorte 1 und $93 \%$ in Kohorte 2 ein unmutiertes IgVH oder eine TP53-Anomalie. Zur Reduktion der Tumorlast wurde Ibrutinib in den ersten drei Zyklen als Monotherapie verabreicht; ab Zyklus 4 wurde zusätzlich Venetoclax in fünf Dosisschritten hochtitriert. Die Venetoclax-Therapie wurde zwei Jahre lang, Ibrutinib bis zum Progress gegeben.

Seit August 2016 starteten 116 Patienten mit der Behandlung. 39 erhalten derzeit noch die Ibrutinib-Monotherapie; die übrigen 77 wurden bereits kombiniert behandelt. Das mediane Follow-up erstreckt sich derzeit über knapp ein Jahr.

Durch die Kombination wurden in beiden Kohorten hohe Raten an Remissionen erreicht, die sich bei längerer Therapiedauer vertieften: Nach der dreimonatigen Ibrutinib-Monotherapie lag die Rate partieller Remissionen (PR) bei den therapienaiven Patienten bei $97 \%$, die Rate an CR und an CR mit unvollständiger hämatologischer Erholung (CRi) bei 3\%. Nach Addition von Venetoclax stieg die CR/CRi-Rate innerhalb eines Jahres kontinuierlich bis auf $100 \%$ an. Zudem wurden alle Patienten innerhalb von zwölf Monaten MRD-negativ. In Kohorte 1 bei rrCLL war das Ansprechen erwartungsgemäß geringer: Nach zwölfmonatiger Kombinationstherapie betrug die CR/CRi-Rate $80 \%$; insgesamt $40 \%$ der Patienten waren MRD-negativ geworden. Bislang wurde im Gesamtkollektiv nur ein Progress verzeichnet; ein Patient starb an einer Cryptococcus-Infektion.
Jain bezeichnete die Toxizität des Zweierregimes als akzeptabel: $44 \%$ der Patienten entwickelten Neutropenien vom Grad 3-4, nur $4 \%$ Thrombozytopenien. Das TLS-Risiko bei gefährdeten Patienten wurde durch den Therapieeinstieg mit Ibrutinib deutlich reduziert. „Damit stellt die Kombination Ibrutinib/Venetoclax eine sichere und effektive Zytostatikafreie Therapie der CLL dar", resümierte Jain.

Weitere Daten zur Sicherheit und Effektivität von Ibrutinib/Venetoclax stammen aus der britischen CLARITY-Studie an 54 rrCLL-Patienten (4). Auch hier wurde die Zweierkombination insgesamt gut vertragen: Die meist gastrointestinalen und hämatologischen Nebenwirkungen bezeichnete Prof. Dr. Peter Hillmen, Leeds, Großbritannien, als vorhersehbar und geringgradig. Ein Patient entwickelte ein TLS, das jedoch durch die verzögerte Gabe von Venetoclax kontrolliert werden konnte und bei späterer Dosissteigerung nicht wieder auftrat.

\section{Prüfung als First-line-Therapie läuft}

Nach achtmonatiger Therapie, davon sechs Monate mit der Kombination, hatten 39\% der Patienten eine $C R$, $8 \%$ eine CRi erreicht. Weitere $53 \%$ sprachen partiell an, sodass sich die Gesamtresponserate auf $100 \%$ summiert. Zudem wurden $37 \%$ der Patienten im peripheren Blut, 32\% im Knochenmark MRD-negativ. Aufgrund der positiven Studiendaten wurde die in Großbritannien laufende Phase-IIIStudie FLAIR zur CLL-Erstlinientherapie bereits modifiziert: Neben den Armen mit Ibrutinib mono und Ibrutinib/Rituximab beinhaltet die Studie jetzt einen weiteren Arm mit der Ibrutinib/Venetoclax-Kombination. Vergleichstherapie im Kontrollarm ist der Standard Fludarabin/Cyclophosphamid/Rituximab (FCR).

\section{Hohe Rate an MRD-Negativität}

In einer Studie der Phase Ib wurde die Kombination von Venetoclax mit dem Anti-CD20Antikörper Obinutuzumab bei 32 therapienaiven CLL-Patienten evaluiert (5). Obinutuzumab wurde zunächst als Monotherapie verabreicht, ab Zyklus 2 Venetoclax in aufsteigender Dosis zugesetzt, berichtete Dr. Ian W. Flinn, Nashville/USA. Auch mit diesem Regime wurde eine Gesamtansprechrate von 100\% erreicht. Die Rate an CR/CRi lag im Gesamtkollektiv bei $72 \%$. Grundsätzlich waren die $\mathrm{CR} / \mathrm{CRi}$-Raten in den verschiedenen zytogenetischen Risikogruppen mit 60-82\% ähnlich hoch, betonte Flinn.

Alle Patienten waren zu mindestens einem Zeitpunkt im Studienverlauf MRD-negativ. Nach drei bis zwölf Monaten nach Therapieende besaßen weiterhin 72-91\% der Teilnehmer einen negativen MRD-Status. Bislang entwickelten drei Patienten im Bobachtungszeitraum von median 18,5 Monaten einen Progress; kein Patient ist verstorben. Der PFS-Median ist noch nicht erreicht; die 18-Monats-Rate beträgt $90,5 \%$.

Bei drei Viertel der Teilnehmer traten Nebenwirkungen vom Grad 3-4 auf. Doch musste Venetoclax nur in einem Fall wegen einer Diarrhö vom Grad 3 abgesetzt werden. Ein klinisch manifestes TLS entwickelte kein Patient. Aufgrund der hohen CR-Rate und der starken MRD-Eradikation sowie der vorläufigen PFSErgebnisse geht Flinn von einer langfristig anhaltenden Effektivität des Zytostatika-freien Obinutuzumab/Venetoclax-Regimes aus.

\section{Innovatives Tripelregime}

Erste Ergebnisse aus einer Phase-II-Studie gibt es außerdem zum Tripelregime mit Obinutuzumab, Ibrutinib und Venetoclax (6). Sie umfasste 25 median 59 Jahre alte Patienten; $71 \%$ besaßen ein unmutiertes IgVH, weitere $24 \%$ einen komplexen Karyotyp. Die Therapie startete in Zyklus 1 mit Obinutuzumab über insgesamt 
acht Zyklen. Ab Zyklus 2 wurde Ibrutinib, ab Zyklus 3 Venetoclax wiederum unter allmählicher Hochtitration hinzugefügt. Insgesamt wurden 14 Zyklen verabreicht, die Patienten danach noch zwei weitere Monate nachbeobachtet. Mittlerweile hat die Hälfte der Patienten die Kombinationstherapie bereits beendet; das Follow-up beträgt median 14,7 Monate.

Auch das Tripelregime zeichnete sich durch eine hohe Responserate von $96 \%$ nach acht Zyklen aus. Fünf Patienten $(20 \%)$ sprachen mit einer CR, weitere 8 (32\%) mit einer CRi an. Zudem war die Kombination sehr effektiv bei der MRD-Elimination: 14/24 untersuchten Patienten $(58 \%)$ waren sowohl im peripheren Blut als auch im Knochenmark MRD-negativ, betonte Dr. Kerry Rogers, Columbus/USA. Sie bezeichnete die Therapie mit dem Tripelregime als sicher: Die Nebenwirkungen waren überwiegend hämatologischer Art, schwere Toxizitäten selten.

Dr. Katharina Arnheim, Freiburg

\section{Literatur}

1. Seymour JF et al. Lancet Oncol 2017; 18: 230-240.

2. Seymour JF et al. ASH 2017, LBA-2.

3. Jain $\mathrm{N}$ et al. ASH 2017, Abstract 429.

4. Hillmen $P$ et al. ASH 2017, Abstract 428.

5. Flinn IW et al. ASH 2017, Abstract 430.

6. Rogers K et al. ASH 2017, Abstract 431.

Quelle: 59. Jahrestagung der American Society of Hematology (ASH) vom 9. bis 12. Dezember 2017, Atlanta/USA

\section{Chronische lymphatische Leukämie Ibrutinib/Rituximab oder Ibrutinib allein - es kommt darauf an}

Patienten mit rezidivierter chronischer lymphatischer Leukämie ( $r C L L)$ oder Hochrisiko-CLL profitieren nicht generell von Rituximab zusätzlich zur Ibrutinib-Therapie. Allerdings induziert die Kombination schnellere Remissionen und führt zu einer stärkeren Eradikation der minimalen Resterkrankung (MRD). Sie könnte daher für Patienten, bei denen ein rasches Ansprechen gefordert ist, sinnvoll sein. Dies ergab eine am MD Anderson Cancer Center in Houston erstellte Phase-II-Studie, die von Dr. Jan A. Burger, Houston/USA auf dem ASH 2017 vorgestellt wurde.

Teilnehmer waren 206 Patienten median 64 Jahre, die Mehrzahl von ihnen $(n=179)$ mit rezidivierter oder refraktärer Erkrankung. Die 27 nicht vorbehandelten Patienten waren aufgrund von 17p-Deletion oder mutiertem TP53 als Hochrisikokollektiv charakterisiert. Alle Teilnehmer wurden randomisiert der IbrutinibMonotherapie oder der Kombination Ibrutinib/ Rituximab zugeteilt, so Burger.

Die Gesamtansprechrate unterschied sich mit $98 \%$ im Ibrutinib- und 100\% im Kombinationsarm praktisch nicht. Die Rate kompletter Remissionen (CR) stieg von $21 \%$ unter Ibrutinib mono auf $28 \%$ mit der Kombination. Auch in den Subkollektiven therapienaiver bzw. rezidivierter Patienten waren die Gesamtansprechraten in beiden Studienarmen ähnlich (93\% vs. $100 \%$ bzw. $99 \%$ vs. $100 \%$ ).
Bei therapienaiven Patienten wurde die CR-Rate durch Ibrutinib/Rituximab allerdings mehr als verdoppelt (50\% vs. $20 \%$ ). Zudem führte die Kombination in dieser Subgruppe zu einer stärkeren MRD-Eradikation im Knochenmark: So wurde keiner der nur mit Ibrutinib, aber $25 \%$ der kombiniert behandelten Patienten im Studienverlauf MRD-negativ (rezidiviert: $6 \%$ vs. $10 \%$ ). Auch war die Zeit bis zum Erreichen einer CR oder einer CR mit inkompletter hämatologischer Erholung im Kombinationsarm nur etwa halb so lang wie mit Ibrutinib mono (11,5 vs. 21,1 Monate; $p=0,032$ ). Das Intervall bis zur Eradikation von CLL-Zellen aus dem Knochenmark war bei kombinierter Therapie ebenfalls deutlich verkürzt. Progressionsfreies und Gesamtüberleben unterschieden sich nach einem Follow-up von median rund zwei Jahren in den beiden Studienarmen jedoch nicht.

Insgesamt 56 Patienten (Ibrutinib mono: $\mathrm{n}=23$; Ibrutinib/Rituximab: $\mathrm{n}=33$ ) brachen die Studie vorzeitig ab, vor allem aufgrund von Nebenwirkungen. Fünf Patienten starben im Therapieverlauf. Die häufigsten Nebenwirkungen waren in beiden Therapiearmen vergleichbar: Hypertonie, Neutropenie, Diarrhö und Vorhofflimmern.

Auf Basis dieser Daten bleibt die Monotherapie mit Ibrutinib bei der CLL Standard, resümierte Burger. Die zusätzliche Gabe eines Antikörpers könne jedoch bei Patienten erwogen werden, bei denen eine rasche Remissionsinduktion wünschenswert ist.

\section{Dr. Katharina Arnheim, Freiburg}

\section{Literatur}

1. Burger JA et al. Randomized Trial of Ibrutinib Versus Ibrutinib Plus Rituximab $(\mathrm{Ib}+\mathrm{R})$ in Patients with Chronic Lymphocytic Leukemia (CLL). ASH 2017, Abstract 427

Quelle: Oral Abstract Session „CLL: Therapy, excluding Transplantation: Targeting MRD Negative CLL through Combinations of Novel Agents and Antibody" im Rahmen der 59. Jahrestagung der American Society of Hematology (ASH) am 10. Dezember 2017, Atlanta/USA. 